\title{
Familial oligodontia and regional odontodysplasia associated with a PAX9 initiation codon mutation
}

\author{
Sari Koskinen $^{1} \cdot$ Riikka Keski-Filppula $^{2,3} \cdot$ Heikki Alapulli ${ }^{4} \cdot$ Pekka Nieminen $^{4} \cdot$ Vuokko Anttonen $^{5,6} \mathbb{C}$
}

Received: 30 June 2018 / Accepted: 12 February 2019 / Published online: 26 February 2019

(C) The Author(s) 2019

\begin{abstract}
Objective Tooth agenesis is one of the most common craniofacial developmental anomalies. In hypodontia, one to five teeth are missing, whereas oligodontia refers to the absence of at least six teeth, excluding the third molars. Mutations in several genes including $M S X 1, P A X 9, A X I N 2$, and WNT10A have been shown to cause non-syndromic tooth agenesis. Regional odontodysplasia (RO), also known as "ghost teeth," is a rare developmental anomaly of tooth formation affecting both dentitions. Some possible causes of RO have been suggested, yet the etiology remains unknown. Because the phenotypes of both oligodontia and RO co-occur in one Finnish family, the aim here was to investigate the genetic etiology of the two conditions. Materials and methods A mutation screening of the genes MSX1, PAX9, AXIN2, and WNT10A was performed for the family members of a RO patient and family history of oligodontia.

Results An initiation codon mutation of the $P A X 9$ gene was found in the proband and segregating with oligodontia in the family. Conclusions The etiology of regional odontodysplasia (RO) may be genetic and the same genes can be involved both in RO and tooth agenesis.

Clinical relevance Our results give new insights into the etiology of regional odontodysplasia, yet further results are needed.
\end{abstract}

Keywords Regional odontodysplasia $\cdot$ RO $\cdot$ Oligodontia $\cdot$ Etiology $\cdot$ PAX9

\section{Introduction}

According to literature, regional odontodysplasia (RO) is a rare odontological disorder which may affect both primary and permanent teeth in one section of the dentition; in about half of the cases, both primary and permanent dentitions are

Vuokko Anttonen

vuokko.anttonen@oulu.fi

1 Department of Oral and Maxillofacial Diseases, Oulu University Hospital, POB 21, 90029 Oulu, Finland

2 PEDEGO Research Unit, Clinical Genetics, Medical Research Center Oulu, University of Oulu, Oulu, Finland

3 Department of Clinical Genetics, Oulu University Hospital, Oulu, Finland

4 Department of Oral and Maxillofacial Diseases, Clinicum, University of Helsinki, Helsinki, Finland

5 Institute of Dentistry, Department of Cariology, Endodontology and Pedodontics, University of Oulu, POB 5281, 90014 Oulu, Finland

6 Medical Research Center, Oulu University Hospital and University of Oulu, POB 21, 90029 Oulu, Finland affected. The condition is more common among females than males. RO may affect dentition in maxilla or mandible commonly involving maxillary lateral incisor regions. $\mathrm{RO}$ affects contiguous teeth in one quadrant, usually not exceeding the midline. Most of the literatures on RO are case reports; in the 2000 s, only a few reviews have been published [1-3].

The etiology of regional odontodysplasia is unclear. Infections, medications, vascular defects, trauma, and nutritional deficiencies have been suggested as etiological factors of the disorder [2,4]. The heritability of the disorder has not been established [2]. Occasionally, RO has been connected with some other disorders, such as vascular naevi, the anomaly of the affected side of the face, hydrocephalus, and hypophosphatasia [5].

Clinically, disturbances in tooth eruption may appear. Both periodontal and periapical infections are associated with RO-swellings, gingivitis, and abscesses. Fibrous tissue covers the alveolar crest. Teeth appear malformed and are often small in size. They may be hypoplastic and/or hypomineralized, and there may also be an increased incidence of agenesis. The color of the teeth is brown or yellow and the tooth enamel is soft and prone to decay. When teeth 
are extracted, the tooth tissue is thin and eggshell-like. Radiographically, teeth with short roots are often called "ghost teeth" due to their shadowy, poorly mineralized appearance concerning both dentin and enamel. The pulp chambers are often wide. Histologically, the enamel prism structure is atypical and contains the so-called apatitic needles and remnants of enamel matrix. In dentin, irregular dentin dominates and the dentin layer is thinner than normal. On the other hand, the thickness of predentin layer is increased. The number and structure of dentinal tubules varies according to the severity of the condition [2].

One of the most common dental disorders is tooth agenesis, i.e., non-syndromic hypo- (one to five teeth), oligo- (more than six teeth), and complete anodontia, prevalence decreasing by the severity of the condition. Congenital tooth agenesis most commonly affects maxillary incisors and mandibular and maxillary premolars. Non-syndromic tooth agenesis has been shown to be hereditary and caused by mutations MSX1, PAX9, AXIN2, WNT10A, EDA, and EDAR genes [6, 7].

The aim of the study was to investigate the genetic etiology of regional odontodysplasia in a patient treated in the Oulu University Hospital, Finland, with a family history of oligodontia.

\section{Material and methods}

\section{Subjects}

The study population comprised a single Finnish family with regional odontodysplasia and tooth agenesis. A pedigree was drawn on the basis of interviews with family members, clinical examinations and available clinical data (Fig. 1).

\section{Mutation detection}

Blood and saliva samples were collected from the proband, her parents, her maternal aunts, and her grandparents for genomic DNA isolation. DNA samples extracted from blood were analyzed for WNT10A (ref. seq. NM_025216.2; NC_000002.11), AXIN2 (ref. seq. NM_004655.3; NC_000017.10), and PAX9 (ref. seq. NM_006194.2; NC_000014.7) genes in the laboratory of UMC Utrecht, the Netherlands. Their DNA test is based on a PCR analysis followed by a sequence analysis of coding exons including flanking "splice-site" consensus sequences.

DNA was isolated from saliva with the Oragene DNA purifier reagent (DNA Genotek, Ottawa, Ontario, Canada) according to the manufacturer's instructions. A mutational analysis of exons of PAX9, MSX1, and WNT10A was performed as described earlier (see Supporting Information Table 1 in Arte et al. 2013) [6].

\section{Ethics}

All participants gave their written informed consent for this case report.

\section{Results}

\section{Phenotype analysis}

A 6-year-old girl was referred from primary health care in the City of Oulu to the Oulu University Hospital, Finland, because of dental developmental disturbances in maxillary right quadrant. The pregnancy and delivery had occurred without any complications. Birth weight was $3815 \mathrm{~g}$ and the Apgar scores were $9 / 10 / 10$. The patient's medical history was clear. At the time of the referral, the growth and development of the patient as well as her skin, nail, hair and eyebrows, and oral soft tissues were normal. No facial asymmetry was discovered. The occlusion was Angle Class I. Horizontal overbite was $3.5 \mathrm{~mm}$ and vertical overbite was $4.5 \mathrm{~mm}$. The patient had a frontal deep bite. Alveolar crest in maxilla was hanging on the affected side.

Clinically, at the age of 6.5 years, there was a partial, delayed eruption and gingival swelling of the permanent right central incisor. The erupted right maxillary primary molars, a canine, and a lateral incisor were all yellow and hypoplastic. There was a fistula scar on the gingiva of the maxillary right lateral primary incisor. The morphology of all other maxillary teeth was normal. The mandibular teeth were morphologically normal and erupting without problems (Fig. 2a).

Panoramic radiograph showed all teeth being affected by $\mathrm{RO}$ in the maxillary right quadrant (Fig. 2b). Ghostly appearance was seen specifically in the region dd. 11-15. The pulps were large and there was lack of contrast between dentin and enamel. The affected teeth were smaller in size and had shorter roots than the rest of the teeth. An orthopantomographic X-ray (OPG) showed no signs of the development of the maxillary second permanent molars or maxillary right premolars. According to the clinical and radiographic findings, the patient was given a diagnosis of regional odontodysplasia associated with agenesis of five permanent teeth (Fig. 2). The treatment of the patient has been and is complex and multidisciplinary, involving an orthodontist, an oral surgeon, and a pediatric dentist. As an example, a removable denture has been presented in Fig. 3.

The mother and her two sisters had numerous missing teeth fulfilling the criteria for oligodontia without any other defects. The mother was missing all premolars and molars. One of her sisters was missing altogether 12 permanent teeth in all quadrants including (dd.17, 16, 15, 13, 12, 25, 26, 27, 37, 31, 41, 47). The other sister was missing 17 teeth also in all quadrants (dd.17, 16, 15, 14, 12, 22, 25, 26, 27, 37, 36, 35, 31, 41, 45, 
Fig. 1 Pedigree of a family with $\mathrm{RO}$ and oligodontia. a The proband (III: 1) has RO and oligodontia. Her mother (II: 1), mother's sisters (II: 2, 3), and grandfather (I: 1) have showed only oligodontia. All affected family members have $P A X 9$ gene c. $2 \mathrm{~T}>$ A mutation. b DNA sequence electropherogram of the proband showing the initiation codon mutation of $P A X 9$ which changes ATG to AAG

Fig. 2 a Clinical appearance of a 6.5-year-old girl with regional odontodysplasia affecting the right maxillary quadrant. b Ortopantomograph showing typical appearance of teeth with regional odontodysplasia (RO) in the right maxillary quadrant a

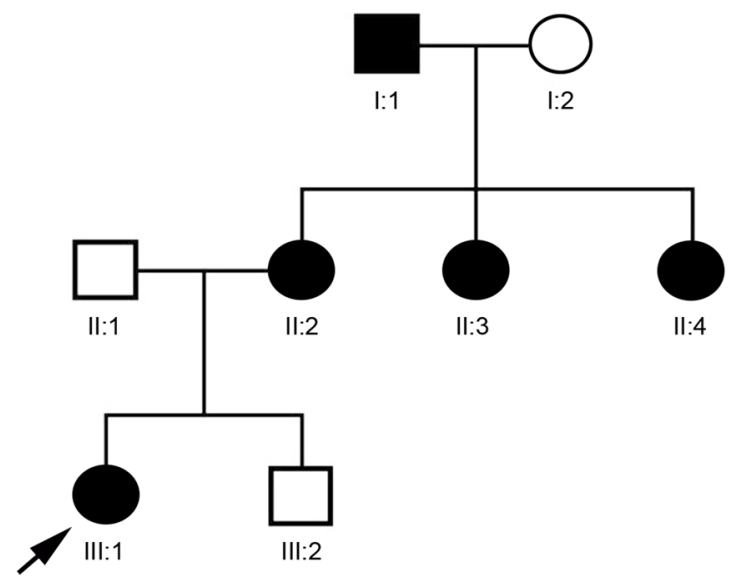

b

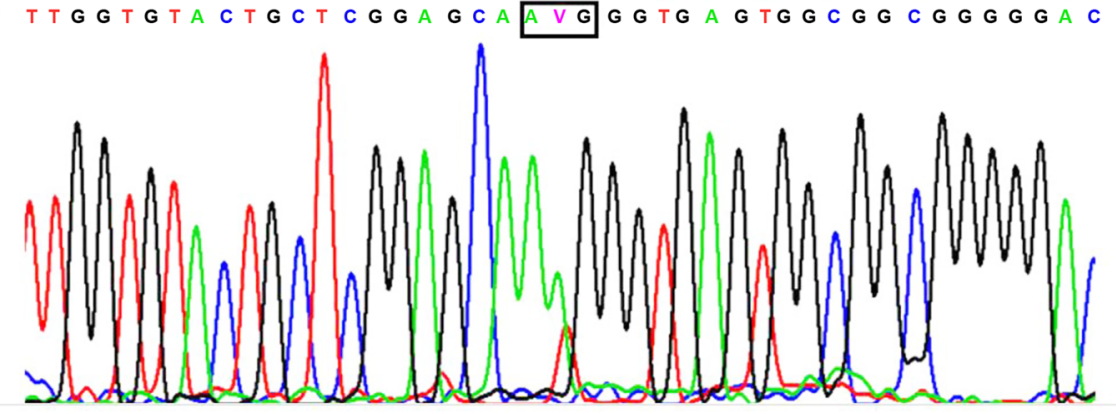

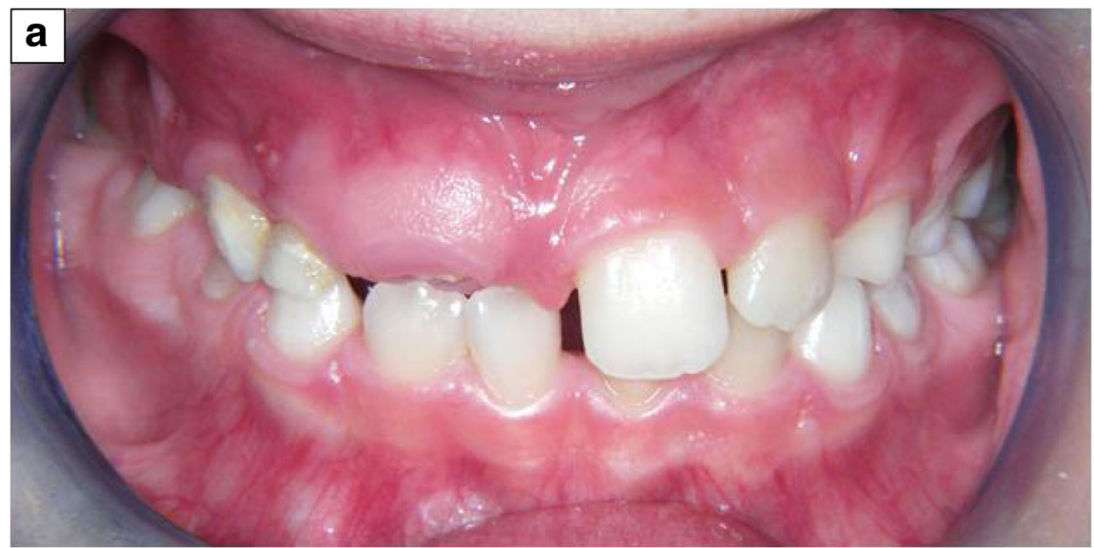

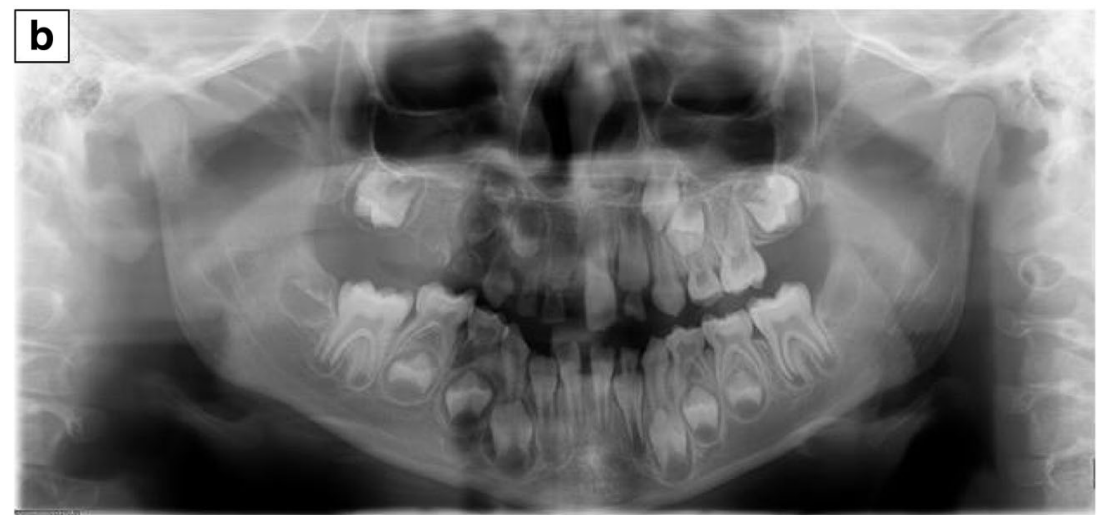


Fig. 3 a Clinical appearance of the patient with regional odontodysplasia at the age of 8 years. b The situation with removable denture
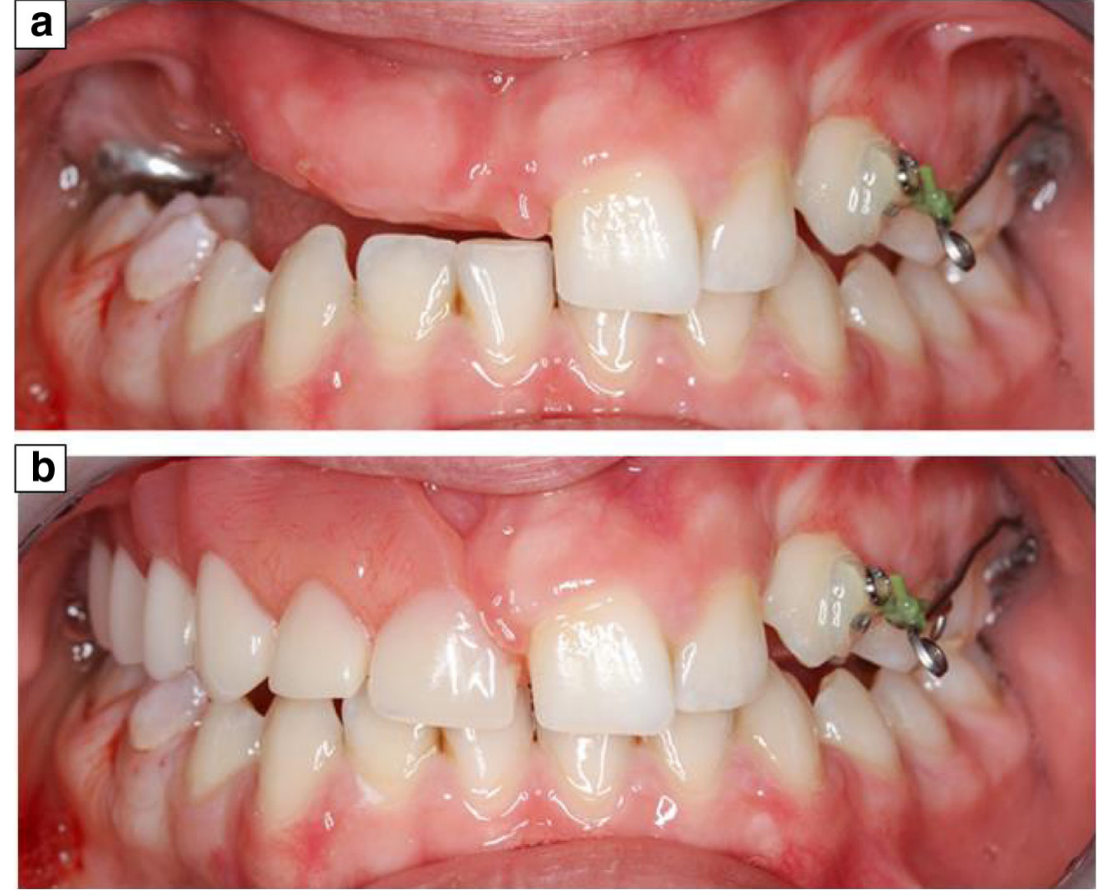

$46,47)$. Without clinical evidence, the mother of the patient reported missing teeth of the grandfather.

\section{Mutation analysis}

A mutation screening of the four genes associated with hypoor oligodontia (WNT10A, PAX9, MSX1, and AXIN2) revealed a heterozygous mutation in $P A X 9$. The NM_006194.2:c.2T>A (NP_006185.1:p.Met1?) variant in exon 2 changes the translation initiation codon ATG to AAG preventing protein production from the mutated copy of the gene. Software analyses used by the UMC laboratory predicted the variant to have pathogenic properties. It has not been observed in control cohorts (ExAC, 1000Genomes) and SIFT and MutationTaster predict this variant to be disease-causing. The variant was also found from the affected mother, the aunts, and the grandfather and it was absent in the sample of the healthy father (Fig. 1).

\section{Discussion}

We describe here a girl with regional odontodysplasia. Interestingly, a pedigree analysis indicated a family history of oligodontia segregating in an autosomal dominant manner. Several genes including MSX1, WNT10A, AXIN2, and PAX9 have been associated with non-syndromic tooth agenesis but the cause of RO is unknown. Mutations in PAXY have been reported in numerous studies and they characteristically most strongly affect molars and premolars, sometimes also deciduous molars [6-8]. The phenotypes of the affected family members in this case confirm this pattern. The identified mutations include three that affect the translation initiation codon, c. $1 \mathrm{~A}>\mathrm{G}, \mathrm{c} .2 \mathrm{~T}>\mathrm{G}$, and c.3G $>\mathrm{A}[7,9,10]$, all expected to cause loss of function as they prevent translation from the affected copy of the gene. As far as we know, this is the first time that a $P A X 9$ gene mutation has been reported in a patient with regional odontodysplasia.

$P A X 9$ is a transcription factor present in dental mesenchyme, activating signals leading to odontogenesis. In mice, lack of $P A X Y$ has been shown to lead to an arrest of odontogenesis at bud stage [11]. Interestingly, incomplete reduction of the $P A X 9$ activity by hypomorphic alleles in mice leads to partial tooth agenesis, enamel defects, and reparative dentin formation [12]. It has been proposed that the inactivation of the Sonic hedgehog $(S h h)$ gene in mice leads to a phenotype that resembles regional odontodysplasia [13]. As $P A X 9$ is known to be necessary for mesenchymal Bmp4 signaling [11], $P A X 9$ deficiency may affect $S h h$ expression in the epithelium. Thus, it is possible that a $P A X 9$ mutation predisposes dental development to an abnormality like regional odontodysplasia. Even though RO-like defects have not been previously described in patients with $P A X 9$ mutations, our finding suggests that special attention should be paid to ROlike features in these patients.

Hypodontia and especially oligodontia require special attention from the dentist, whereas RO is rare and challenging for a clinician. In any case, early diagnosis is necessary. 3D technology is a novel modern instrument for studying the structure of the tissues surrounding the tooth [14]. Knowledge on the etiology and genetics of RO facilitates the early diagnosing of the condition, which again is essential 
for treatment planning. A multidisciplinary approach is necessary when making a treatment plan. In treatment planning, an orthodontist, a surgeon, and even a prosthodontist can be of most help to a pediatric dentist.

Iizava et al. (2010) suggest retaining RO-affected teeth if possible [15]. Crowns, for instance, stainless steel crowns with ceramics not requiring preparation, have been shown to keep the teeth vital even for several years [16]. However, removing the infected, fragile teeth is often the only option for treatment and even that requires the skills of an experienced surgeon due to their eggshell-like consistency. It must also be borne in mind, that even the bone can be affected by RO. Removing the teeth may also lead to resorption of the alveolar bone, which in a growing child requires continuous care for retaining the occlusal planes. Autotransplantation is a choice for treatment if there is crowding in the dentition in other segments. Implants are a modern way of replacing absent teeth when a person reaches adulthood. If a whole quadrant is affected by RO, like in this case, dentures must be made and adjusted at the phase of the child's growth to maintain the occlusion and the appearance (Fig. 3).

This is the first time that a $P A X 9$ gene mutation has been found from a patient with regional odontodysplasia or RO. So far, the etiology of RO remains unknown. Our findings suggest that there may be a common etiology for tooth agenesis and $\mathrm{RO}$, but further research is needed to establish the role of $P A X 9$ in these processes. Increasing genetic testing of dental patients and the use of exome/genome wide analyses will give us more information about the etiology of this rare disease in the future.

Acknowledgements Open access funding provided by University of Oulu including Oulu University Hospital.

Funding The work was supported by the Department of Oral and Maxillofacial Surgery, Special Dental Care and Orthodontics of Erasmus Medical Centre in Rotterdam, the Netherlands.

\section{Compliance with ethical standards}

Conflict of interest The authors declare that they have no conflict of interest.

Ethical approval All procedures were part of the normal protocol in treating patients with regional odontodysplasia and severe oligodontia. All procedures involving human participants were in accordance with the ethical standards of the institutional research committee of Northern Ostrobothnia Hospital District and with the 1964 Helsinki declaration.

Informed consent Informed consent was obtained from all participants.

Open Access This article is distributed under the terms of the Creative Commons Attribution 4.0 International License (http:// creativecommons.org/licenses/by/4.0/), which permits unrestricted use, distribution, and reproduction in any medium, provided you give appropriate credit to the original author(s) and the source, provide a link to the Creative Commons license, and indicate if changes were made.
Publisher's note Springer Nature remains neutral with regard to jurisdictional claims in published maps and institutional affiliations.

\section{References}

1. Hamdan MA, Sawair FA, Rajab LD, Hamdan AM, Al-Omari IK (2004) Regional odontodysplasia: a review of the literature and report of a case. Int J Paediatr Dent 14:363-370

2. Tervonen SA, Stratmann U, Mokrys K, Reichart PA (2004) Regional odontodysplasia: a review of the literature and report of four cases. Clin Oral Investig 8:45-51

3. Al-Tuwirqi A, Lambie D, Seow WK (2014) Regional odontodysplasia: literature review and report of an unusual case located in the mandible. Pediatr Dent 36:62-67

4. Kappadi D, Ramasetty PA, Rai KK, Rahim AM (2009) Regional odontodysplasia: an unusual case report. J Oral Maxillofac Pathol 13:62-66. https://doi.org/10.4103/0973-029X.57671

5. Crawford PJ, Aldred MJ (1989) Regional odontodysplasia: a bibliography. J Oral Pathol Med 18:251-263 Review. Erratum in J Oral Pathol Med 18: following 425

6. Arte S, Parmanen S, Pirinen S, Alaluusua S, Nieminen P (2013) Candidate gene analysis of tooth agenesis identifies novel mutations in six genes and suggests significant role for WNT and EDA signaling and allele combinations. PLoS One 22:e73705. https:// doi.org/10.1371/journal.pone.0073705 eCollection 2013

7. Liang J, Qin C, Yue H, He H, Bian Z (2016) A novel initiation codon mutation of PAX9 in a family with oligodontia. Arch Oral Biol 61: 144-148. https://doi.org/10.1016/j.archoralbio.2015.10.022

8. Stockton DW, Das P, Goldenberg M, D’Souza RN, Patel PI (2000) Mutation of PAX9 is associated with oligodontia. Nat Gen 24:18-19

9. Klein ML, Nieminen P, Lammi L, Niebuhr E, Kreiborg S (2005) Novel mutation of the initiation codon of PAX9 causes oligodontia. J Dent Res 84:43-47

10. Sarkar T, Bansal R, Das P (2017) A novel G to A transition at initiation codon and exon-intron boundary of PAX9 identified in association with familial isolated oligodontia. Gene 635:69-76. https://doi.org/10.1016/j.gene.2017.08.020

11. Peters H, Neubuser A, Kratochwil K, Balling R (1998) Pax9-deficient mice lack pharyngeal pouch derivatives and teeth and exhibit craniofacial and limb abnormalities. Genes Dev 12:2735-2747

12. Kist R, Watson M, Wang X, Cairns P, Miles C, Reid DJ, Peters H (2005) Reduction of Pax9 gene dosage in an allelic series of mouse mutants causes hypodontia and oligodontia. Human Mol Gen 14: 3605-3617

13. Spini TH, Sargenti-Neto S, Cardoso SV, Souza KC, de Souza SO, de Faria PR, Lyola AM (2007) Progressive dental development in regional odontodysplasia. Oral Surg Oral Med Oral Pathol Oral Radiol Endod 104:e40-e45

14. Matsuyama J, Tanaka R, Iizawa F, Sano T, Kinoshita-Kawano S, Hayashi-Sakai S, Mitomi T (2014) Clinical and radiographic findings and usefulness of computed tomographic assessment in two children with regional odontodysplasia. Case Rep Dent 2014:1-5. https://doi.org/10.1155/2014/764393

15. Iizawa F, Kinjoh N, Taguchi Y (2010) Regional odontodysplasia: long-term observation of a case on the mandibular left side. Pediatr Dent J 20:103-109

16. Mostafa M, Taha N, Mehrez M (2013) Generalized versus regional odontodysplasia: diagnosis, transitional management, and longterm follow-up - a report of two cases. Case Rep Dent 2013:1-5. https://doi.org/10.1155/2013/519704 